\title{
Variación fenotípica de la concha en Neritinidae (Gastropoda: Neritimorpha) en ríos de Puerto Rico
}

\author{
Juan Felipe Blanco ${ }^{1}$, Silvana Tamayo ${ }^{1}$ \& Frederick N. Scatena ${ }^{2,3}$ \\ 1. Instituto de Biología, Facultad de Ciencias Exactas y Naturales, Universidad de Antioquia, Calle 67 \# 53-108, \\ Medellín, Colombia. Apartado Aéreo 1226; blanco@exactas.udea.edu.co; silvatamaqui@gmail.com \\ 2. Departamento de Ciencias de la Tierra y Ambientales, Universidad de Pensilvania, Filadelfia, Estados Unidos. \\ 3. Fallecido
}

\author{
Recibido 12-XII-2013. Corregido 20-I-2014. Aceptado 13-II-2014.
}

\begin{abstract}
Phenotypic variability of the shell in Neritinidae (Gastropoda: Neritimorpha) in Puerto Rican rivers. Gastropods of the Neritinidae family exhibit an amphidromous life cycle and an impressive variability in shell coloration in Puerto Rican streams and rivers. Various nominal species have been described, but Neritina virginea [Linné 1758], N. punctulata [Lamarck 1816] and N. reclivata [Say 1822] are the only broadly reported. However, recent studies have shown that these three species are sympatric at the river scale and that species determination might be difficult due to the presence of intermediate color morphs. Individuals (8 751) were collected from ten rivers across Puerto Rico, and from various segments and habitats in Mameyes River (the most pristine island-wide) during three years (2000-2003), and they were assigned to one of seven phenotypes corresponding to nominal species and morphs (non-nominal species). The "axial lines and dots" morph corresponding to $N$. reclivata was the most frequent island-wide, while the patelliform $N$. punctulata was scant, but the only found in headwater reaches. The "yellowish large tongues" phenotype, typical of $N$. virginea s.s. was the most frequent in the river mouth. The frequency of secondary phenotypes varied broadly among rivers, along the rivers, and among habitats, seemly influenced by salinity and predation gradients. The occurrence of individuals with coloration shifts after predation injuries, suggests phenotypic plasticity in the three nominal species, and urges for the use of molecular markers to unravel the possible occurrence of a species complex, and to understand the genetic basis of polymorphism. The longitudinal distribution of individual sizes, population density and egg capsules suggested the adaptive value of upstream migration, possibly to avoid marine predators. Rev. Biol. Trop. 62 (Suppl. 2): 53-68. Epub 2014 April 01.
\end{abstract}

Key words: Neritina virginea, Neritina reclivata, Neritina punctulata, shell color, phenotypic variability, polymorphism.

Los gasterópodos de la familia Neritinidae (Gastropoda: Neritimorpha) tienen una gran variabilidad fenotípica asociada con la salinidad, la cual ha sido escasamente estudiada en el Caribe (Bandel, 2001). Uno de los grandes desafíos en el estudio de las poblaciones de los Neritinidae es la identificación de los individuos, debido al gran número de especies nominales simpátricas, tal como se ha observado en la región del Pacífico (Haynes, 1993; Tan \& Clements, 2008). En el caso de los Neritinidae del Caribe, Neritina virginea [Linneo 1758] y
N. punctulata [Lamarck 1816] son claramente diferenciadas por la forma de su concha (Bandel, 2001), pero varias especies nominales fueron propuestas posteriormente e incluidas en guías de campo y listados de especies generando gran confusión (Russell, 1941; Warmke \& Abbott, 1961; Aguayo, 1966). Esto pudo ser causado por polimorfismos o incluso plasticidad fenotípica equivalente a la observada en especies de otras regiones (Pacífico americano: Valdéz \& Barrantes, 1980; Indopacífico: Grüneberg, 1976, 1978, 1979). 
Las especies del género Neritina presentan una distribución tropical y subtropical, y son abundantes en las costas, estuarios y ríos del Caribe (Humfrey, 1971; Abbott, 1974; Díaz \& Puyana, 1994). Exhiben un ciclo de vida anfídromo (McDowall, 2007), que consiste en el desarrollo de diferentes etapas de vida alternadas en los ámbitos dulciacuícola y marino, presentando, por lo tanto, migraciones entre ambos (Crandall, 1999; Blanco \& Scatena, 2006). Debido a los desplazamientos río arriba de los individuos reclutas y juveniles, que les llevan toda su vida (Shigemiya \& Kato, 2001; Schneider \& Lyons, 1993; Schneider \& Frost, 1986; Pyron \& Covich, 2003), las poblaciones se extienden varios kilómetros río arriba (Blanco \& Scatena, 2006; Resh, Barnes \& Craig, 1990). Esto también genera una segregación longitudinal de tallas, encontrándose individuos más pequeños principalmente en zonas bajas y cercanas a la desembocadura, mientras que los adultos predominan en las zonas altas (Shigemiya \& Kato, 2001; Schneider \& Lyons, 1993; Schneider \& Frost, 1986; Pyron \& Covich, 2003). El comportamiento migratorio de los más pequeños es posiblemente causa de la alta probabilidad de ser depredados (Schneider \& Lyons, 1993; Blanco \& Scatena, 2005; 2007; Blanco \& Arroyave, 2009).

Neritina virginea y $N$. punctulata se encuentran desde Florida y Bahamas hasta el Norte de Brasil y desde el golfo de México y América Central hasta Las Antillas. Son simpátricos en las partes bajas de los ríos y quebradas, pero mientras los primeros predominan desde las planicies costeras hacia la desembocadura, e inclusive en las praderas de fanerógamas y los manglares, los segundos predominan en los segmentos montañosos. El tamaño oscila entre 4 y $25 \mathrm{~mm}$, desde los individuos recientemente reclutados hasta los adultos más grandes. Información detallada sobre la ecología de $N$. virginea en quebradas costeras del Caribe ha sido publicada por Blanco \& Scatena (2005, 2006, 2007) y Blanco \& Arroyave (2009). Sobre $N$. punctulata existe menos información debido a que solo es abundante en las cabeceras de las quebradas y ríos costeros del Caribe, aunque habita de manera simpátrica y en bajas densidades con $N$. virginea en las partes bajas (Pyron \& Covich, 2003; Blanco \& Scatena, 2006). A pesar de las diferencias morfológicas de la concha de ambas especies su ecología es similar (Blanco \& Scatena, 2006).

Neritina virginea s.s. $(4-15 \mathrm{~mm})$ se caracteriza por una concha globular y delgada con color de fondo variable (verde, rosa o amarillo), con líneas axiales oscuras y manchas claras con un borde resaltado en negro. El ámbito de variación de las manchas o puntos va desde puntos irregularmente distribuidos hasta escamas triangulares y lenguas de gran expansión hacia abajo de la sutura, regularmente distribuidos. Una o dos bandas espirales blancas se observan en algunos individuos, lo cual también fue observado en nerítidos del Indopacífico (Grüneberg, 1976). Neritina clenchi y $N$. piratica [Russell, 1940] se proponen como sinónimos de $N$. virginea (Díaz \& Puyana, 1994; Bandel, 2001). Neritina clenchi presenta un color naranja oscuro o fondo rojo, y más cerca de los puntos de sutura, y $N$. piratica presenta un patrón de líneas apretadas negras que forman una red reticulada de forma triangular o de diamante. A pesar de ser simpátricas, sólo $N$. reclivata [Say 1822] y $N$. punctulata son actualmente reconocidas como especies diferentes de $N$. virginea en la mayoría de los catálogos (Humfrey, 1971; Abbott, 1974; Díaz \& Puyana, 1994), pero dado que no existen descripciones de las partes blandas y análisis genéticos tal distinción es aun dudosa. La concha de $N$. reclivata es de color marrón oliva oscuro con numerosas líneas finas axiales de color negro, marrón o lavanda, pero en ocasiones su degradación de color y características radulares degradan en $N$. virginea (Bandel, 2001). La concha de N. punctulata es distintivamente grande (hasta $25 \mathrm{~mm}$ ), en forma pateloide, con manchas circulares uniformemente espaciadas sobre un fondo verde oliva. Estas dos últimas especies son consideradas aparte de $N$. virginea por los coleccionistas aficionados. El pie de los Neritidae de agua dulce (2-4 mm) es de color oscuro a marrón claro, y no presenta 
una coloración adulta distintiva en cualquier especie nominal.

En "complejos de especies" como este, es necesario explorar, primero, la existencia de polimorfismos $\mathrm{y}$, finalmente, la plasticidad fenotípica. Mientras el polimorfismo ha sido observado en varios Neritinidae (e.g. Valdéz \& Barrantes, 1980), la plasticidad fenotípica solo ha sido formalmente sugerida para Clithon oualaniensis [Lesson, 1831] de la región Indo-Pacífica (Grüneberg, 1976, 1978, 1979). La plasticidad fenotípica es la capacidad de un solo genotipo para producir diferentes fenotipos en ambientes distintos (Hollander \& Butlin, 2010). Los diferentes colores de las conchas de algunos Neritidae y otros gasterópodos son un ejemplo de plasticidad fenotípica que ha generado problemas con la delimitación adecuada de especies (Minton, Norwood \& Hayes, 2008). Esta plasticidad se ha atribuido a factores como el estrés térmico (Hazel \& Johnson, 1990), exposición al oleaje (Brown \& Quinn, 1988), densidad de población (Kemp \& Bertness, 1984) y depredación (Grüneberg, 1976; Appleton \& Palmer, 1988; Sepúlveda, Jara \& Gallardo, 2012). En las conchas de gasterópodos se han identificado diversas respuestas de plasticidad tales como el grosor (Hoverman \& Relyea, 2007; Minton et al., 2008), el grosor y la ornamentación (Brookes \& Rochette, 2007), la producción de pigmentos (Palma \& Steneck, 2001; Manríquez, Lagos, Jara \& Castilla, 2009) a lo largo de gradientes ambientales y de depredación. En los Neritidae intermareales Puperita pupa [Linnaeus, 1767] y P.tristis [Orbigny, 1842] se han encontrado diversos patrones de plasticidad de la coloración en respuesta a la posición intermareal, la temperatura y salinidad (Minton \& Gunderson, 2001), mientras que en los Neritidae de agua dulce se han observado diferencias en el grosor del opérculo y de la concha en el gradiente agua salada-dulce (Vermeij \& Covich, 1978), al punto que la variación de la dureza de las conchas y formación de "alas" de acuerdo al hábitat ha sido observado en Neritina grano$s a$ [Sowerby, 1825] en quebradas de Hawaii (Ford, 1979).
Para abordar el problema del polimorfismo en Neritina spp. de Puerto Rico, como una etapa inicial para explorar la posible plasticidad fenotípica, se plantearon los siguientes objetivos de estudio: 1) identificar las frecuencias de los patrones de coloración y forma predominantes, 2) evaluar la distribución espacial de dichos patrones fenotípicos entre hábitats y segmentos a lo largo del curso de un río (i.e., Mameyes) y entre varios ríos alrededor de la isla, y 3) describir la distribución longitudinal de la densidad de la población, tallas de los individuos y la oviposición, como una primera aproximación al valor adaptativo de la migración río arriba.

\section{MATERIALES Y MÉTODOS}

Área de estudio: El estudio se realizó en la isla caribeña de Puerto Rico, cuyo clima es marítimo tropical debido a que la precipitación durante la época de lluvias es influenciada por frentes fríos y depresiones tropicales. $\mathrm{La}$ mayor precipitación $(\geq 300 \mathrm{~mm} / \mathrm{mes})$ ocurre en los periodos abril-mayo y agosto-diciembre. Debido a que la isla es casi rectangular y tiene las mayores elevaciones en la cordillera Central y la Sierra de Luquillo, todos los ríos son considerados costeros, ya que atraviesan una región montañosa y luego la planicie costera. Una descripción más amplia del área de estudio se encuentra en Blanco \& Scatena $(2005 ; 2006)$.

Distribución de los fenotipos: Entre agosto de 2000 y julio de 2003, se llevaron a cabo muestreos esporádicos en tres hábitats (una charca marginal, charcas dentro de su cauce y rápidos) en un segmento de $400 \mathrm{~m}$ aguas arriba y abajo del puente de la carretera estatal No. 3 de Puerto Rico sobre el río Mameyes (descrito en Blanco \& Scatena, 2005; 2006; 2007; Blanco \& Arroyave, 2009). El número de fechas de muestreo varió de 6 (charca marginal) a 63 (charcas dentro del cauce), para un total de 116 muestreos. Entre 100 y 500 individuos fueron seleccionados al azar dentro de 10-20 cuadros de muestreo también dispuestos al azar dentro de cada hábitat 
en cada fecha. Además, durante el verano de 2001 y 2002, se llevaron a cabo muestreos en la desembocadura del río en el mar, en el estuario, en la planicie costera $(<20 \mathrm{~m})$ y en varias zonas montañosas (baja: $<50 \mathrm{~m}$, media: 50-100m, alta: $>100 \mathrm{~m}$ ) en los ríos Guajataca, Arecibo, Manatí, La Plata, Loíza, Espíritu Santo, Mameyes, Fajardo, Patillas y Nigua, y un total de 8751 individuos fueron recogidos: 6971 en el río Mameyes entre 2000 y 2003 y 1780 en todos los ríos de la isla (incluyendo el Mameyes) durante el verano de 2001 y 2002.

Los individuos fueron clasificados en los siete fenotipos que representan todo el espectro de variación en las especies nominales de Neritina, también observados en otros Neritinidae del Indopacífico por Grüneberg (1976) (Fig. 1): 1) líneas axiales (=N. reclivata s.s.), 2) líneas axiales y puntos o lenguas $(=N$. virginea s.s.), 3) grandes lenguas expandidas desde la sutura ( $=N$. virginea s.s), 4) bandas espirales blancas $(=N$. virginea $), 5)$ fondo negro y reticulado $(=N$. virginea $), 6)$ color de fondo naranja o rojizo ( $=N$. virginea), y 7) concha pateliforme, verde olivo con manchas blancas $(=N$. punctulata). Se calcularon los porcentajes de frecuencia media para cada fenotipo y se utilizaron pruebas Chi cuadrado para determinar la uniformidad de fenotipos dentro y entre los hábitats, y entre sitios a lo largo del curso del río (Sokal \& Rohlf, 1995).

Variación del tamaño individual, la densidad poblacional y la oviposición a lo largo del río Mameyes: Tres muestreos de un solo día (10 de octubre 2000, 21 de junio 2001, 13 de julio 2003) se llevaron a cabo en el río Mameyes para establecer la densidad y el

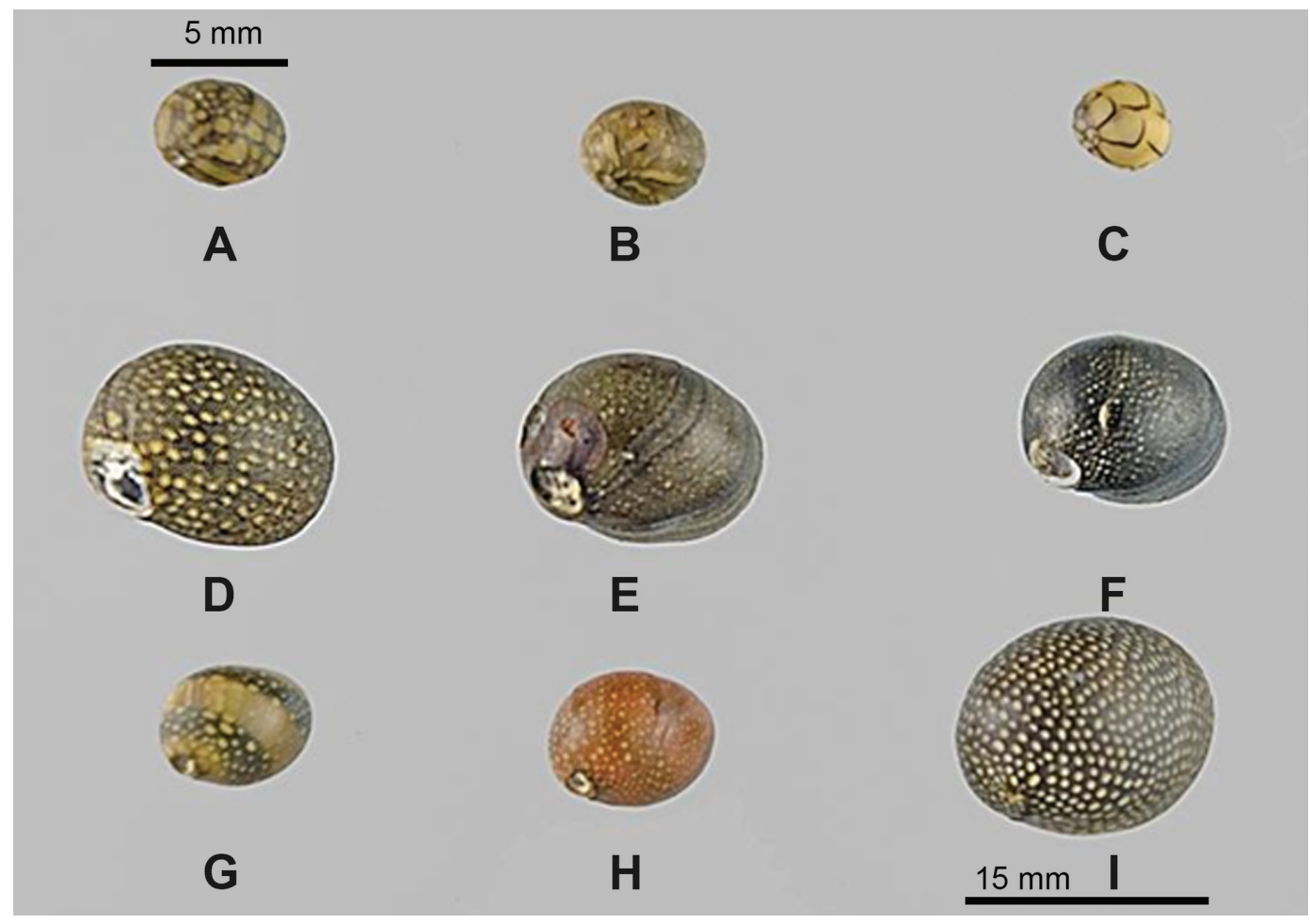

Fig. 1. Variación del color de la concha de Neritinidae en Puerto Rico. A, B y C: lenguas grandes. D: líneas axiales y puntos. E: líneas axiales. F: negra. G: bandas espirales. H: rojiza. I: pateloide. La escala sobre la concha A aplica para las demás, excepto la I.

Fig. 1. Color variation in the shell of Neritinidae in Puerto Rico. A, B and C: large tongues, D: axial lines and dots. E: axial lines. F: black. G: spiral bands. H: redish. I: patelloid. Scale line in A applies to all shells, but I. 
tamaño de los individuos en ocho sitios entre la desembocadura del río y una altura de 50 msnm (el sitio más alto al que se puede acceder por carretera). Los sitios fueron: Estuario, en la playa arenosa en la desembocadura del río (estación 1, Playa Fortuna), en los humedales y manglares en el estuario bajo (estación 2), entre los jacintos de agua flotantes en el estuario medio (estación 3); Planicie costera, entre el campo de golf del Club Westin Riomar, y en la bocatoma de Palmer administrada por la Autoridad Puertorriqueña de Acueductos y Alcantarillados, AAA (estaciones 4 y 5); Montaña baja, correspondiente al sector conocido como La Vega (estación 6); Montaña alta, correspondiente al segmento entre Puente Roto (estación 7) y el sendero Angelito (estación 8) (Fig. 2). La densidad fue determinada mediante el conteo de los individuos bajo el agua en $10-20$ cuadrados $(50 \times 50 \mathrm{~cm})$ colocados aleatoriamente en varios hábitats. El ancho de las conchas se midió utilizando un pie de rey digital o calibrador $(0.01 \mathrm{~mm})$. Debido a que la densidad de cápsulas ovígeras depositadas sobre

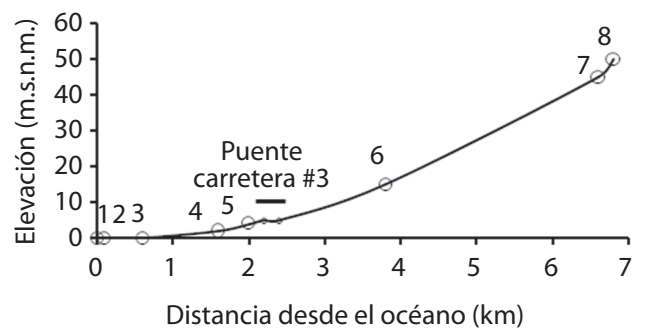

Fig. 2. Gradientes longitudinal y de elevación en el río Mameyes en los que se muestrearon la densidad de individuos, ancho de la concha, largo promedio de las cápsulas ovígeras y número promedio de huevos por cápsula. Estaciones: 1 y 2: Playa Fortuna y estuario bajo, 3: estuario medio, 4 y 5: campo de golf (planicie costera), 6: La Vega (montaña baja), 7: Puente Roto, 8: Sendero Angelito (montaña alta). Se muestra el cruce de la Carretera \#3 sobre el río.

Fig. 2. Longitudinal and elevation gradients in Mameyes river where individual density, shell width, egg capsule length, and number of eggs were sampled. Stations: 1 and 2: Playa Fortuna and lower estuary, 3: mid estuary, 4 y 5: golf course (planicie costera), 6: La Vega (lower montane), 7: Puente Roto, 8: Angelito's Trail (upper montane). The location of the Route \#3 Bridge over the river is shown. las rocas es muy variable, se tomó la longitud de las mismas y el número de embriones dentro de cada una como un estimativo de la fecundidad a lo largo del río. Durante la última fecha de muestreo, se recolectaron cinco rocas que fueron transportadas en un recipiente con agua al laboratorio, donde se midieron las cápsulas con un micrómetro en el estéreo microscopio $\mathrm{y}$, posteriormente, se extrajeron los embriones utilizando una aguja hipodérmica con alcohol $70 \%$. El contenido fue depositado en una caja de Petri y los embriones fueron contados utilizando el estereoscopio. El tamaño de los individuos (ancho de la concha), la densidad media de la población, el largo de las cápsulas ovígeras y el número de embriones dentro de las cápsulas, fue comparado entre sitios a lo largo del curso del río Mameyes mediante un Análisis de Varianza de Una Vía (ANDEVA) y las diferencias fueron evaluadas mediante una prueba post hoc de Tukey (Zar, 1999).

\section{RESULTADOS}

Patrones entre hábitats del río Mameyes (Fig. 3): Dentro de los 6971 individuos recolectados en el río Mameyes, la frecuencia promedio de fenotipos presentó variaciones entre hábitats. El fenotipo de líneas axiales y puntos presentó la mayor frecuencia, pero esta fue mayor en la charca marginal del río $(68 \%)$ y menor en los rápidos $(59 \%)$. Entre los fenotipos secundarios, el negro tuvo menor frecuencia en charcas marginales $(5 \%)$ que en rápidos $(10 \%)$, mientras que el pateloide fue más frecuente en charcas que en rápidos. Los fenotipos con lenguas grandes y bandas espirales presentaron una frecuencia similar entre hábitats. A pesar de las leves diferencias, las frecuencias de fenotipos secundarios tuvieron la misma proporción en todos los hábitats $\left(\chi^{2}=7.055 ;\right.$ g.l. $\left.=10 ; p=0.720\right)$.

Patrones longitudinales en los ríos alrededor de la isla (Fig. 4): Dentro de los 1780 individuos recolectados en varios ríos alrededor de la isla, se encontró que los individuos con líneas axiales y puntos fueron los más 
A

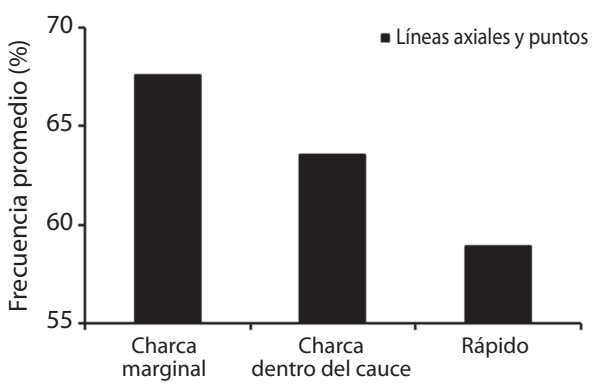

B

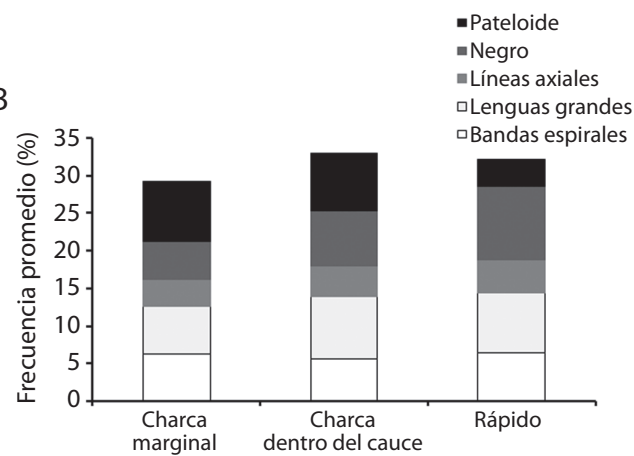

Fig. 3. Frecuencia de fenotipos en varios hábitats en el segmento de la planicie costera del río Mameyes. El fenotipo líneas axiales y puntos se muestra en el panel A y los demás en el B. Número de individuos colectados entre agosto de 2000 y julio de 2003: Charca marginal (208), charcas dentro del cauce (4874) y rápido (1 889). Número total de individuos: 6971 .

Fig. 3. Phenotype frequency in various habitats in a coastal plain segment in the Mameyes River. The "axial lines and dots" phenotype in shown in panel A while the rest are shown in panel B. Habitat names and number of individuals collected between August 2000 and June 2003: High flow pond (208), instream pools (4874), and riffle (1889). Total number of individuals: 6971. Phenotype names: Axial lines and dots, large tongues, axial lines, spiral bands, black, and patelloid.

frecuentes a lo largo de diferentes segmentos de los ríos, especialmente en el estuario medio con un $89 \%$. Este fue seguido por el fenotipo de lenguas grandes, que aunque estuvo presente en varios segmentos de los ríos, su frecuencia fue mayor en el estuario bajo (27\%) y la desembocadura (18\%) aunque ausente en el estuario medio. Los individuos con bandas espirales presentaron una distribución longitudinal similar, estando igualmente ausentes en el estuario medio y una mínima frecuencia en la

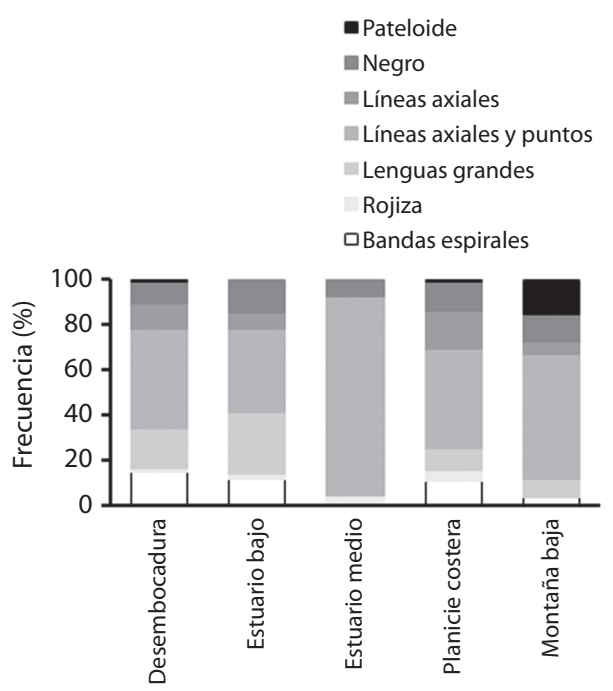

Fig. 4. Frecuencia de fenotipos a lo largo de varios ríos alrededor de Puerto Rico. Número de individuos recolectados en el verano de 2001 y 2002: desembocadura (474), estuario bajo (689), estuario medio (27), planicie costera (356) y montaña baja (132). Número total: 1780 (102 individuos no fueron asignados a ningún fenotipo debido a que no se encontraba diferenciado su color debido al pequeño tamaño).

Fig. 4. Phenotype frequency along various rivers in Puerto Rico. Segment names and number of individuals collected during summer in 2001 and 2002: river mouth (474), lower estuary (689), mid estuary (27), coastal plain (356), lower montane (132). Total number: 1780 (102 individuals were not assigned to a phenotype due to their undifferentiated color and small size). Phenotype names: Axial lines and dots, large tongues, axial lines, spiral bands, black, redish, and patelloid.

montaña baja. Los fenotipos negros y de líneas axiales fueron constantes en su distribución longitudinal, aunque no se encontraron estos últimos en el estuario medio. El fenotipo rojizo fue constante pero poco frecuente a lo largo del río. El fenotipo pateloide presentó una baja frecuencia y estuvo prácticamente restringido a la zona de montaña baja en los ríos Mameyes, Espíritu Santo, Sabana, Arecibo y Patillas. Existió una asociación entre cada fenotipo encontrado y cada zona de distribución longitudinal $\left(\chi^{2}=342.9 ;\right.$ g.l. $\left.=15 ; \mathrm{p}<0.001\right)$.

Patrón de frecuencias de fenotipos alrededor de la isla y en el río Mameyes (Fig. 5): Dentro de los 1780 individuos recolectados 


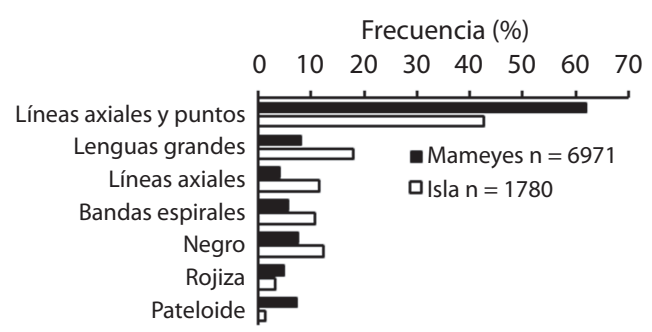

Fig. 5. Frecuencia de fenotipos alrededor de la isla de Puerto Rico (verano 2001 y 2002) y en el río Mameyes (2000-2003).

Fig. 5. Phenotype frequency island-wide in Puerto Rico (summer 2001 and 2002) and at Mameyes River (20002003). Phenotype names: Axial lines and dots, large tongues, axial lines, spiral bands, black, redish, and patelloid.

alrededor de la isla, el fenotipo predominante fue el de líneas axiales y puntos con una frecuencia de $43 \%$. Los fenotipos de lenguas grandes, líneas axiales y negros presentaron una frecuencia entre el 10 y $20 \%$, mientras que los fenotipos pateloide y de fondo rojizo presentaron 2 y $3 \%$, respectivamente.

El río Mameyes fue el mejor muestreado ( $n=6971$ individuos) en términos de hábitats, recorrido longitudinal y número de visitas (fechas). Por lo tanto, en él se registraron todos los fenotipos hallados alrededor de la isla, siendo clara la predominancia del fenotipo de líneas axiales y puntos (62\%), seguido de lenguas grandes $(8 \%)$; los demás fenotipos fueron secundarios $(<8 \%)$. Sin embargo, fue notorio que algunos fenotipos secundarios estuvieron sub- o sobre-representados en este río.

Variación de la frecuencia de los fenotipos secundarios entre varios ríos de la isla (Fig. 6): En 2001 y 2002, la proporción de los fenotipos secundarios varío dentro de cada río $\left(\chi^{2}=233.598\right.$; g.l. $\left.=32 ; \mathrm{p}<0.001\right)$. Por ejemplo, en Arecibo y Mameyes el fenotipo rojizo fue más escaso $(1 \%)$ que en los otros ríos, y en Espíritu Santo fue más frecuente (8\%); en La Plata el fenotipo líneas axiales presentó baja frecuencia (4\%) y en Mameyes estuvo ausente, mientras que en los otros ríos ocupó el segundo o tercer lugar en frecuencia; en Espíritu Santo el fenotipo lenguas grandes fue el más escaso (6\%), mientras que en Mameyes fue el más frecuente (42\%); en Guajataca y Patillas el fenotipo bandas espirales fue poco frecuente (3 y $8 \%$, respectivamente), pero en Nigua fue predominante (17\%). Aunque el fenotipo pateloide no se incluyó en este análisis de frecuencias, fue dominante, pero en bajas densidades, en la parte montañosa de los ríos Mameyes y Espíritu Santo, mientras que en Arecibo, Patillas y Sabana fueron muy escasos (1 ó 2 individuos) y en los restantes ríos estuvieron ausentes. Otros ríos en los que se recolectaron algunos caracoles en la desembocadura fueron: Blanco (4), Ceiba (12), Fajardo (13) y Manatí (11). En los manglares de bahía de Jobos (5) y de la isla de Vieques (6) se encontraron algunos caracoles.

Cambios de coloración durante el crecimiento de los individuos (Fig. 7): En cerca de un $10 \%$ de los individuos se observaron cambios de coloración correspondientes a dos o más fenotipos a lo largo del eje principal de expansión o crecimiento de la concha. En su mayoría, estos cambios estuvieron asociados con la presencia de una marca de depredación sobre el antiguo borde de la concha. El cambio de color se presentó sobre la nueva porción de concha generada a partir del borde roto. En algunos casos excepcionales, los cambios de un fenotipo a otro no estuvieron mediados por una marca de depredación, ni alteraciones visibles en las líneas de crecimiento, sin embargo, esto se observó predominantemente en conchas $<5 \mathrm{~mm}$. Se observaron cambios entre todos los fenotipos, sin embargo, los cambios desde o hacia el fenotipo pateliforme ( $N$. punctulata) solo se observaron en individuos $<5 \mathrm{~mm}$.

Variación la densidad poblacional, el tamaño individual y la oviposición a lo largo del río Mameyes (Fig. 8): La densidad promedio de individuos disminuyó progresivamente a medida que se ascendió por la montaña $\left(\mathrm{F}_{5,91}=24.6, \mathrm{P}<0.001\right)$, en contraste, con el ancho promedio de las conchas $\left(\mathrm{F}_{7,780}=554.3\right.$, $\mathrm{P}<0.001)$. El largo promedio de las cápsulas ovígeras y el número de embriones en ellas 
Frecuencia (\%)

Frecuencia (\%)
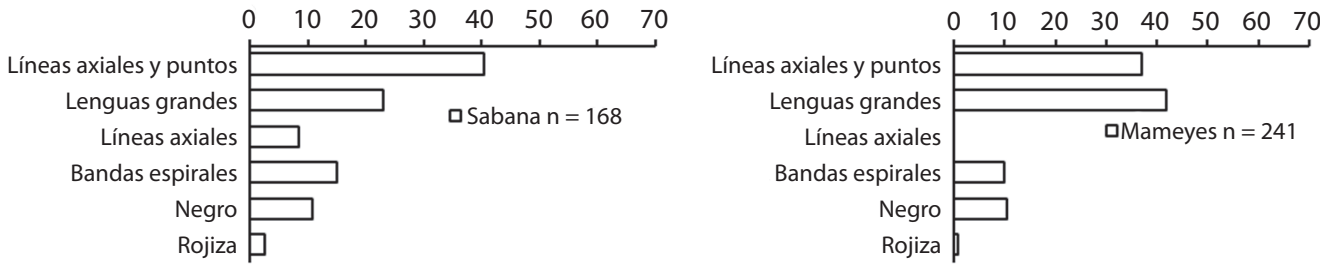

Frecuencia (\%)

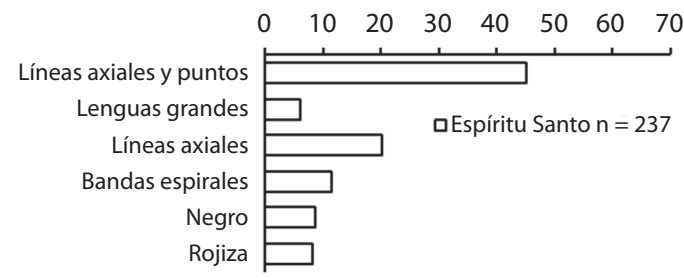

Frecuencia (\%)

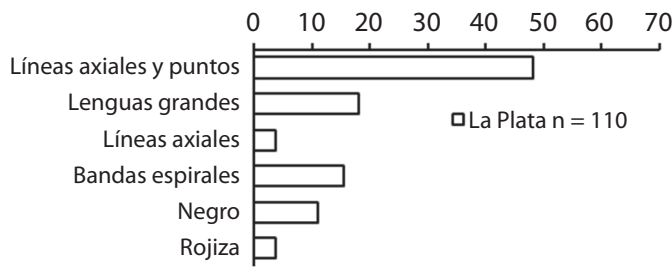

Frecuencia (\%)

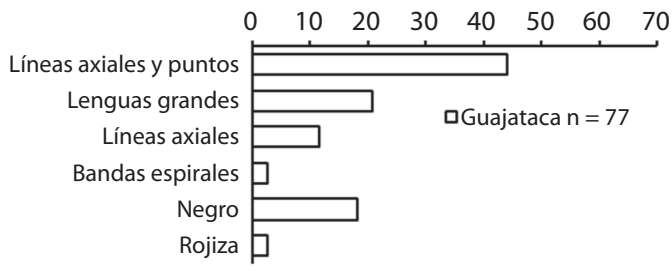

Frecuencia (\%)
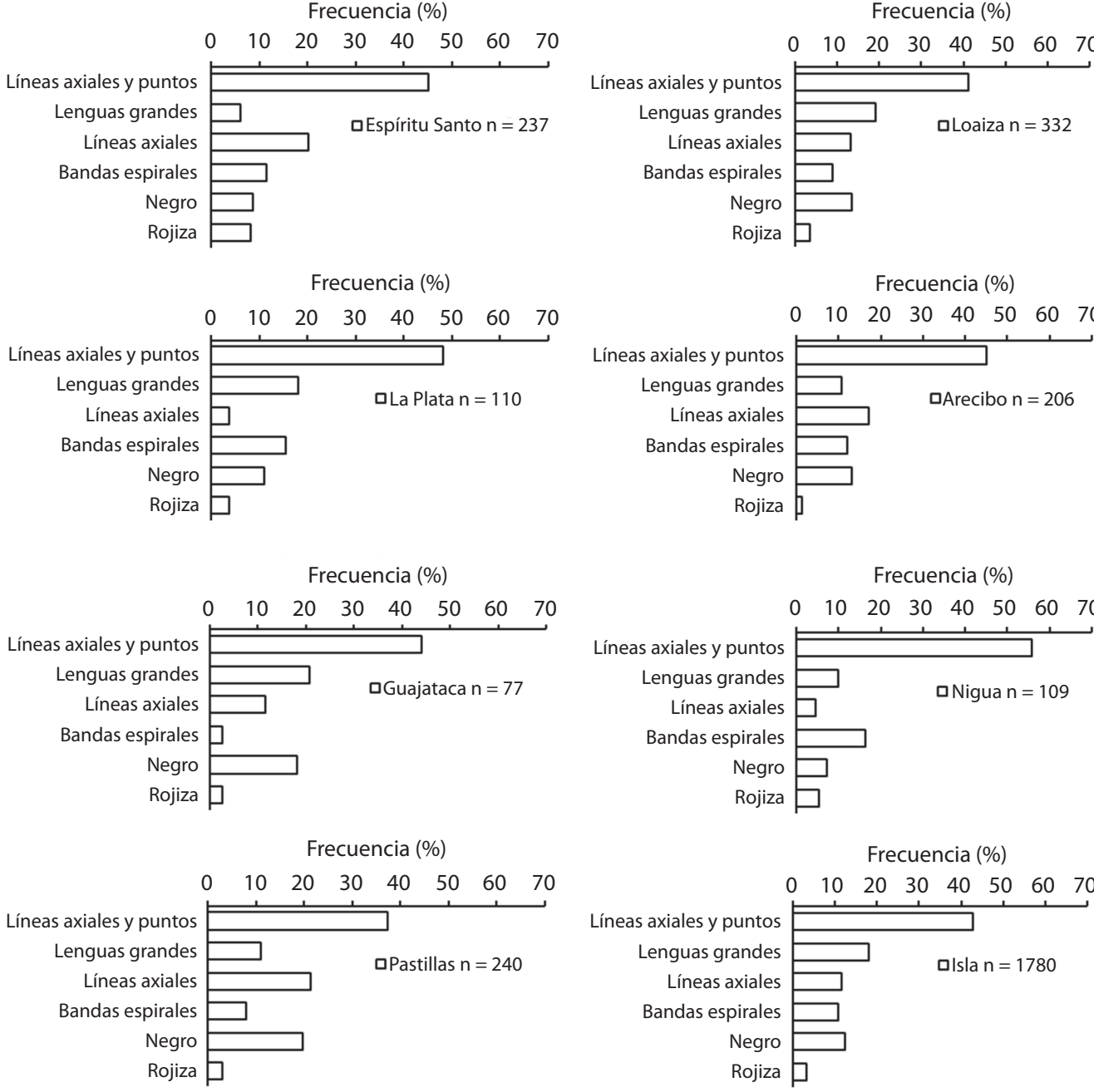

Frecuencia (\%)

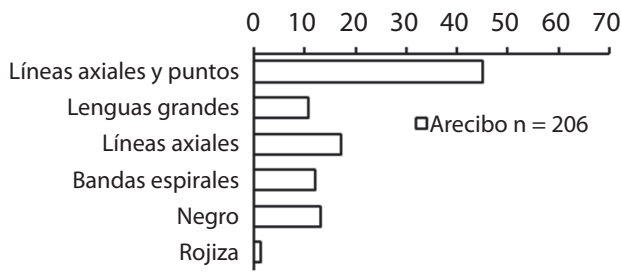

Frecuencia (\%)

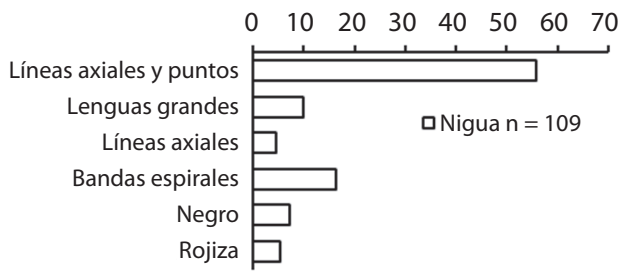

Frecuencia (\%)

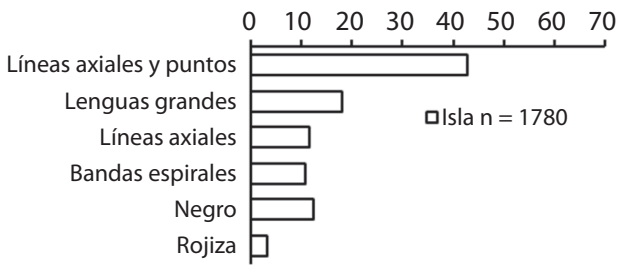

Fig. 6. Frecuencia de fenotipos en varios ríos de la isla de Puerto Rico. Se excluyó el pateloide porque solo tuvo una abundancia relativa significativa en los ríos Mameyes y Espíritu Santo. Nótese que los datos del río Mameyes en esta figura solo corresponden a los muestreos realizados durante el verano en 2001 y 2002. Se indican los números de individuos por río.

Fig. 6. Phenotype frequency in various rivers of Puerto Rico. The pateloid phenotype was excluded because its relative abundance was only significant in rivers Mameyes and Espíritu Santo. Note that data from Mameyes river only correspond to the samplings during summer 2001 and 2002. Number of individuals per river are shown. Phenotype names: Axial lines and dots, large tongues, axial lines, spiral bands, black, and redish. 


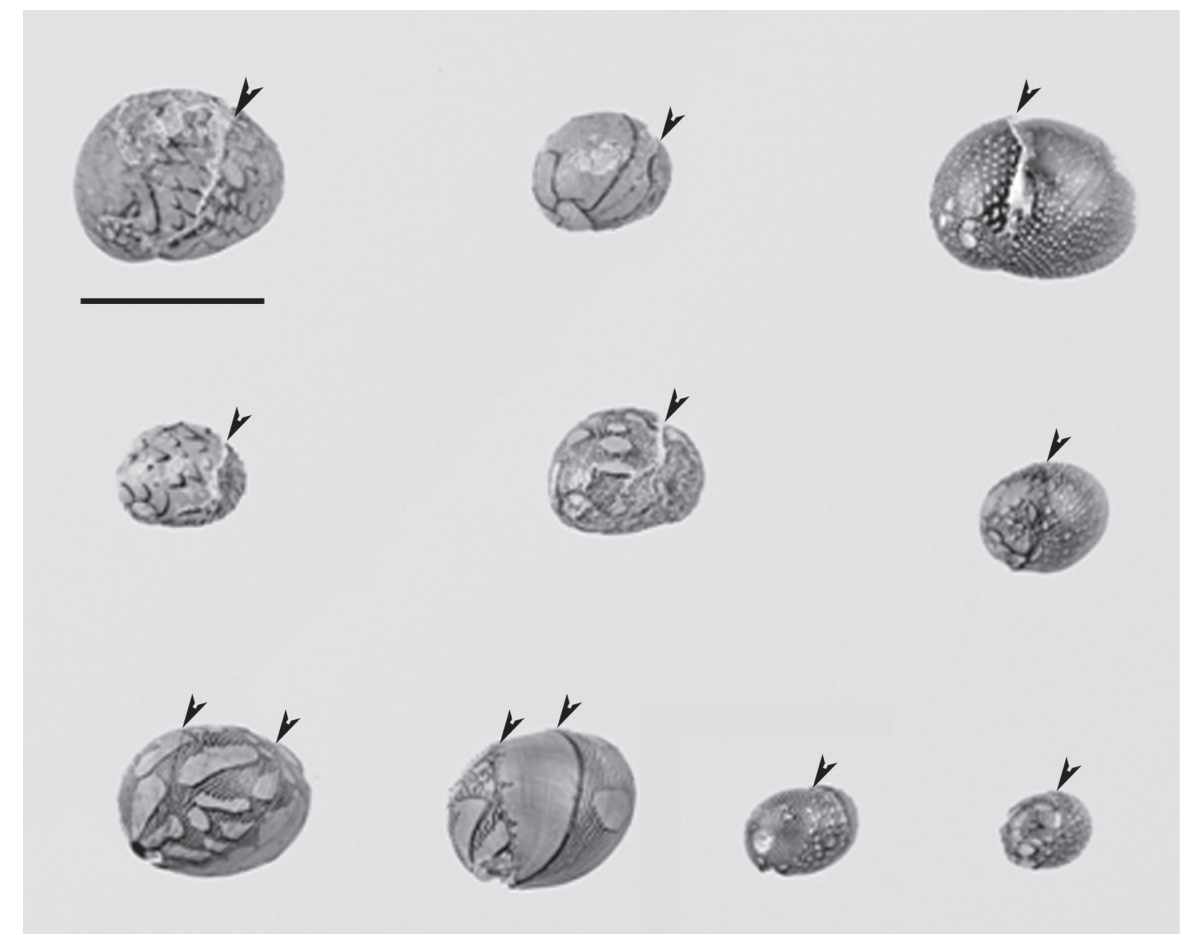

Fig. 7. Cambios de coloración en individuos de Neritina virginea en Puerto Rico. Nótese la coincidencia de los cambios con presencia de marcas de depredación (señalado con flechas). Escala=10 mm.

Fig. 7. Coloration changes in Neritina virginea in Puerto Rico. Note the coincidence of color change with predation marks (pointed by arrows). Scale: $10 \mathrm{~mm}$.

aumentó a partir de la estación 4, donde comienzan a aparecer sobre las rocas (en las estaciones 5 y 8 no se muestrearon) (largo de las cápsulas: $\mathrm{F}_{2,98}=68.1, \mathrm{p}<0.001$; embriones por cápsula: $\left.\mathrm{F}_{2,98}=41.2, \mathrm{p}<0.001\right)$. A pesar del muestreo incompleto del gradiente longitudinal, las cápsulas más grandes y con mayor número de embriones tendieron a aumentar hacia la parte alta de la montaña (Log[embriones por cápsula $]=1.09+1.97 ; \log [$ largo de cápsula $]+0.12$; $\left.\mathrm{r}^{2}=60.7 \%, \mathrm{~F}_{1,99}=155.5, \mathrm{p}<0.001\right)$.

\section{DISCUSION}

Variabilidad fenotípica entre hábitats. La variación de la presión por depredación encontrada por Blanco \& Arroyave (2009) utilizando marcas en las conchas, podría explicar algunas diferencias fenotípicas entre hábitats. En varios segmentos a lo largo del río
Mameyes, los fenotipos negros y de puntos rojizos fueron más frecuentes en rápidos que en charcas donde podrían ser menos conspicuos ante los depredadores. Por otra parte, los pateloides fueron encontrados en menor proporción en aguas poco profundas y rápidos. Lo primero podría explicarse, por el hecho de que aunque los individuos grandes son más frecuentes en las zonas someras, pueden ser depredados por aves (Blanco \& Arroyave, 2009). Lo segundo podría explicarse por el mayor esfuerzo cortante o estrés hidráulico que se produce en los individuos de mayor tamaño (Blanco \& Scatena, 2006; 2007). Los pateloides son usualmente más grandes que los otros fenotipos. En las zonas más profundas podría ocurrir una menor depredación por aves, mientras que en los rápidos podría ocurrir una menor de decápodos, esto debido a que en aguas de baja velocidad y someras la frecuencia de conchas depredadas 

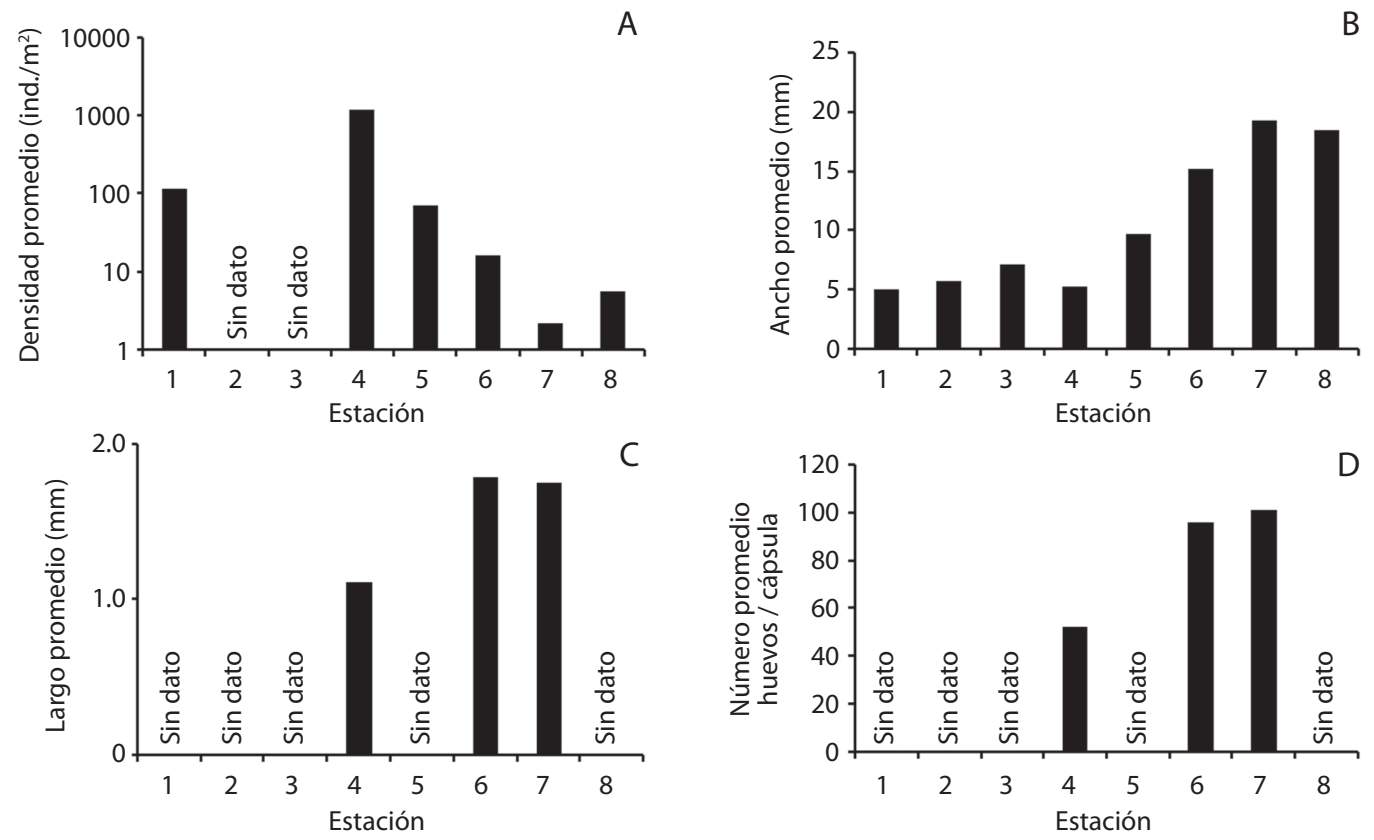

Fig. 8. Variación longitudinal de: A. densidad promedio de individuos, B. ancho promedio de la concha, C. largo promedio de las cápsulas ovígeras y D. número promedio de huevos por cápsula en ocho estaciones a lo largo del río Mameyes (Puerto Rico). Estaciones: 1 y 2: Playa Fortuna y estuario bajo, 3: estuario medio, 4 y 5: campo de golf, 6: La Vega, 7: Puente Roto, 8: Sendero Angelito. Se muestra el cruce de la Carretera \#3 sobre el río. En las estaciones 2 y 3 no fue posible establecer la densidad dado que no se pudo muestrear el fondo, solo la vegetación flotante. En las estaciones 5 y 8 no se muestrearon las cápsulas ovígeras. S.D.: Sin dato.

Fig. 8. Longitudinal variation in: A. mean individual density, B. shell width, D. egg capsule length, and D. number of eggs in eight stations along the Mameyes river (Puerto Rico). Stations: 1 and 2: Playa Fortuna and lower estuary, 3: mid estuary, 4 y 5: golf course, 6: La Vega, 7: Puente Roto, 8: Angelito's Trail. The location of the Route \#3 Bridge over the river is shown. Snail density was not estimated in stations 2 and 3 because the muddy bottom was not sampled, only the floating vegetation. Egg capsules were not sample in stations 5 and 8. S.D.: No data.

es mucho mayor (Blanco \& Scatena, 2005; 2007; Blanco \& Arroyave, 2009).

Finalmente, al poner los cambios de coloración observados (Fig. 7) en contexto de variabilidad de la presión por depredación, la plasticidad fenotípica tendría un valor adaptativo. La migración aguas arriba a través de diferentes hábitats y segmentos del río impone presiones selectivas variables que no pueden ser afrontadas por un solo fenotipo. Por lo tanto, cambios en coloración podrían esperarse a lo largo del crecimiento de las conchas y con los cambios de hábitat. Estos cambios y el polimorfismo de color pueden sugerir la ocurrencia de una sola especie o máximo dos. Es posible que el "complejo" de N. virginea corresponda a una sola especie. Sin embargo, se observaron con frecuencia individuos cambiando de color o forma progresivamente de $N$. virginea a $N$. punctulata a lo largo del río. En $P$. pupa y $P$. tristis, se encontraron evidencias genéticas que demuestran que son la misma especie a pesar de las diferencias fenotípicas de la coloración de la concha (Minton \& Gunderson, 2001). Experimentos de traslado entre hábitats y de exposición a depredación o señales químicas de depredadores podrían demostrar empíricamente la plasticidad fenotípica en los Neritinidae.

Variabilidad fenotípica a lo largo de los ríos y entre ellos: La migración río arriba y la ocupación de diversos hábitats durante 
la misma, expone a los individuos a diferentes presiones selectivas que podrían mantener el polimorfismo de coloración observado en los Neritinidae. En el caso de Puerto Rico, aunque las condiciones físico-químicas y de hábitat de cada río son diferentes (Blanco \& Scatena, 2006) y las proporciones de igual manera varían (en este estudio), se observó un patrón general en todos: $N$. virginea (fenotipo de líneas axiales y puntos $=N$. reclivata) es la especie con mayor frecuencia de individuos, mientras que $N$. punctulata (fenotipo pateloide) se encuentra en una proporción mucho menor y restringida a las partes montañosas principalmente en dos ríos (Mameyes y Espíritu Santo). Este mismo patrón ocurre en otras partes del Caribe insular y continental (J. F. Blanco, observaciones personales).

Se observaron diferencias de las frecuencias de los fenotipos secundarios entre ríos que pueden obedecer a múltiples factores, tales como la concentración de algunos iones y la presión por depredación, como se ha observado en otros Neritinidae. Se ha encontrado que los diversos patrones de coloración en Clithon oualaniensis en el Indo-Pacífico pueden estar influenciados por variables oceanográficas a gran escala, como concentraciones iónicas de los mares, mientras que los cambios individuales pueden ser atribuidos a la inducción directa o indirecta de depredadores (Grüneberg, 1976; 1978; 1979). En Theodoxus fluviatilis [Linné 1758] de Alemania se ha observado que leves cambios en la concentración salina favorecen la producción de pigmentos (Neumann, 1959; Zettler, Frankowski, Bochert \& Rohner, 2004). En N. latissima [Broderip, 1833] del Pacífico costarricense también se han encontrado cinco morfotipos influenciados por las concentraciones de manganeso en los ríos (Valdez \& Barrantes, 1980). Experimentalmente se ha comprobado que el nerítido intermareal Puperita pupa en el Caribe, luego de varios meses de haberse trasladado a charcas de agua dulce, comenzó a tomar la coloración de otra especie nominal de agua dulce, $P$. tristis, comprobando ser finalmente la misma especie respondiendo plásticamente a variaciones de salinidad
(Minton \& Gunderson, 2001). En Puerto Rico, se ha observado que la conductividad y el $\mathrm{pH}$ cambian significativamente entre ríos dependiendo de si su origen es volcánico o cársico (Blanco \& Scatena, 2006), lo cual podría explicar las diferencias en frecuencias de fenotipos secundarios. Un experimento de transplante entre ríos de diferente geología o pruebas de crecimiento bajo diferentes condiciones ambientales en laboratorio podría establecer si el polimorfismo de coloración es un carácter plástico o fijo. Grüneberg (1976) sugirió que en los cambios de color de C. oualaniensis hay sistemas que producen pigmento blanco y pigmento oscuro (leuco-sistema y melano-sistema, respectivamente), estos sistemas dependen de la presencia de activadores y de la velocidad de difusión de elementos antagonistas. Para varias familias de caracoles, particularmente en Neritinidae, se ha descrito matemáticamente que una modificación de las condiciones ambientales conduce a la activación o inhibición de dos o más genes, expresados en cambios en el patrón y en el color de sus conchas lográndose una compleja predicción algorítmica (Meinhardt, 2003).

La significativa variación de la salinidad entre el mar, el estuario, y el río podría explicar las diferencias fenotípicas observadas en el río Mameyes. En este, fue claro que las bandas espirales y las lenguas grandes fueron los fenotipos más abundantes en las playas del estuario (Playa Fortuna), lo cual podría ser influenciado por la concentración de iones particulares o por tipo de arena. Dado que este río es el más conservado de la isla (Blanco \& Scatena, 2006), este patrón puede estar exento de la influencia humana sobre la calidad físico-química del agua.

Si bien los cambios en la concentración de salinidad parecen ser factores relevantes en la distribución de los patrones de coloración longitudinal, la presión por depredadores también podría desencadenar expresiones de plasticidad o distribución diferencial de fenotipos a lo largo del gradiente mar-estuario-río (Grüneberg, 1976; Appleton \& Palmer, 1988; Sepúlveda et al., 2012). En el caso de los Neritinidae 
de Puerto Rico, los fenotipos bandas espirales y lenguas grandes con una concha predominantemente amarilla (correspondientes a $N$. virginea s.s.) se camuflan mejor con la arena coralina que otros fenotipos, pudiendo experimentar un menor riesgo de ser depredados. Pero si el evento de depredación ocurre, el escape es la principal defensa contra la perforación de las conchas, lo cual es consistente con la resistencia a la subyugación y la alta frecuencia de reparación que se observa frecuentemente en Neritinidae desde latitudes templadas a tropicales (Andrews, 1935). El resultado de un evento de depredación fallida es una cicatriz irregular que claramente se aparta del curso normal de las líneas de crecimiento como producto de mecanismos antidepredadores de la especie (Vermeij, 1982). Esta presión por depredación es una de las fuerzas selectivas más fuertes observadas en los gasterópodos de todo el mundo (Vermeij \& Covich, 1978; Vermeij, 1982; Covich, 2010). Aunque en nuestro estudio no se cuantificó la tasa de depredación o la ocurrencia de plasticidad fenotípica, fue observado que un $8 \%$ de las conchas observadas poseían fracturas del labio, posiblemente infligidas por un depredador, que habían sido reparadas con capas nuevas pero con una variación en el patrón de coloración. Este proceso se ha reportado en el gasterópodo marino Acanthina monodon [Pallas, 1774] de Chile, que en pruebas de laboratorio incrementa el grosor de la concha desde la primera fase de una inducción por depredadores (Sepúlveda et al., 2012).

Los depredadores pueden desencadenar la expresión defensiva de la presa, sea a través de la depredación directa o a través de señales químicas (Appleton \& Palmer, 1988; Trussell \& Nicklin, 2002; Sepúlveda et al., 2012). Aunque los cangrejos son los depredadores más comunes de los caracoles en diversas partes del mundo (junto con las aves y los peces), se ha debatido si por ejemplo los cangrejos pueden ver colores o no, ya que por lo general localizan sus presas por el tacto o el olfato o reaccionan más fuertemente a los movimientos, sombras u objetos contrastantes (Pettitt, 1975). Hasta el momento algunas especies de cangrejos parecen tener visión de colores, y si este es el caso, las longitudes de onda de la luz reflejada de las conchas podrían ser relevantes. Sin embargo, los cangrejos depredadores del caracol intermareal Littorina saxatilis en Suecia seleccionan sus presas independientemente del color (Ekendahl, 1998). En este caso, son los peces los más importantes para la selección, razón por la que $L$. saxatilis tiene mayor frecuencia de conchas de colores llamativos en zonas rocosas fuera del alcance de los peces (Johannesson \& Ekendahl, 2002). Por lo tanto, en ausencia de depredadores la producción de plasticidad, polimorfismos y el mantenimiento de defensas que no son necesarios pueden evitarse (DeWitt, 1998).

La incidencia de depredadores como aves y crustáceos en la parte baja del río Mameyes es menor en comparación a ecosistemas como los arrecifes y playas rocosas, a pesar de que los decápodos de agua dulce, depredan ocasionalmente a $N$. virginea a lo largo de todo el río (Blanco \& Arroyave, 2009). Entre los fenotipos observados en el río Mameyes, el pateloide aumenta su frecuencia desde la planicie costera hacia la montaña baja donde aparecen los decápodos dulceacuícolas. Este presenta una mayor dureza de las conchas y a lo largo de todo su ámbito de distribución tiene una baja incidencia de marcas de depredación (J. F. Blanco, observaciones personales). De hecho, en la parte montañosa no se han encontrado conchas de individuos vivos o muertos depredadas por cangrejos o camarones. Por lo tanto, en los estuarios de los ríos, la depredación podría ser una fuerza evolutiva considerable que produce respuestas adaptativas como la migración río arriba de juveniles que poseen las tallas más vulnerables, como lo han hipotetizado Blanco \& Scatena (2005). En N. latissima del Pacífico costarricense también se han encontrado cinco morfotipos que experimentan tasas de depredación diferentes (Valdéz \& Barrantes, 1980).

Posible valor adaptativo de las migraciones río arriba: El aumento del tamaño promedio de los individuos y la reducción de la densidad de los Neritinidae río arriba en 
Puerto Rico, se suman a las migraciones en masa reportadas anteriormente como evidencia del ciclo de vida anfídromo (Pyron \& Covich, 2003; Blanco \& Scatena, 2005; 2006). Sin embargo, este estudio aporta nuevas evidencias que sugieren que los individuos migran aguas arriba para reproducirse, dado que se encontraron mayores tamaños de las cápsulas ovígeras y un mayor número de huevos por cápsula en las estaciones más alejadas del mar. Esto genera una correlación indirecta que sugiere que los individuos más grandes producen un mayor esfuerzo reproductivo, lo cual le podría otorgar un valor adaptativo a la migración río arriba. Shigemiya \& Kato (2001) encontraron que los Neritinidae de Japón presentan un mayor número de copulas a medida que aumenta la distancia desde el mar, como lo sugieren las bolsas espermáticas, concluyéndose que las hembras más longevas copulan más veces. Si a esto se le suman las evidencias directas e indirectas que muestran que los individuos migran río arriba para escapar de los depredadores (Schneider \& Frost, 1986; Schneider \& Lyons, 1993; Blanco \& Arroyave, 2009), la migración adquiere un valor adaptativo, y la depredación una relevancia como presión selectiva. Recientemente, Kano (2009) informó que los individuos de una especie de Neritinidae se suben sobre las conchas de otras, posiblemente como una estrategia para migrar río arriba más rápidamente.

En conclusión, los Neritinidae de Puerto Rico penetran varios kilómetros río arriba durante su ciclo de vida anfídromo y, por ello, atraviesan múltiples paisajes fluviales. Poseen un fenotipo dominante y varios secundarios que varían significativamente en sus frecuencias entre ríos alrededor de la isla, entre segmentos a lo largo del río, y entre hábitats, y hay algunos indicios de que la coloración de la concha sea un carácter plástico. Sin embargo, la variación espacial de las frecuencias fenotípicas y la plasticidad podría estar relacionada con una suma de factores abióticos (e.g. características físico-químicas del agua) y bióticos (e.g. depredación) que interactúan a lo largo de la ontogenia y crecimiento de los individuos durante su ciclo de vida anfídromo. Esta situación es equivalente al "seudo-polimorfismo" identificado por Grüneberg (1982) en el nerítido $C$. oualaniensis del Indopacífico. Por lo tanto, se requieren estudios evolutivos que busquen establecer la causa última de la migración y de la morfología de las conchas, mediante observaciones a lo largo de gradientes ambientales y de experimentos de traslados recíprocos. Se recomienda una revisión sistemática del grupo para descartar la existencia de un "complejo de especies crípticas" y para eliminar las especies nominales descritas con base en caracteres exclusivamente morfológicos de las conchas "vacías". Durante este trabajo se revisaron las muestras y se visitaron los mismos sitios de estudio de Mestey-Villamil (1979) y Pyron \& Covich (2003) en Puerto Rico y por lo tanto se resolvieron los problemas de identificación mencionados en los mismos. Se recomienda para estudios ecológicos posteriores el uso de $N$. virginea y $N$. punctulata como únicas especies nominales con base en las diferencias morfológicas de la concha y a sus patrones de distribución levemente segregados a lo largo de los ríos del Caribe, y se recomienda incorporar $N$. reclivata a la primera debido a la gran variabilidad fenotípica que incluye transiciones hacia y desde la misma, y a que son completamente simpátricas en ríos y manglares. Urge la resolución de su identidad por medio de marcadores moleculares y análisis de morfología de las partes blandas y descartar la ocurrencia de especies crípticas o zonas de hibridización.

\section{AGRADECIMIENTOS}

Este trabajo constituye parte de la disertación doctoral del primer autor y fue financiado por un acuerdo de colaboración (00-CA-11120101-004) entre el Instituto Internacional de Dasonomía Tropical del Servicio Forestal de los Estados Unidos y la Universidad de Puerto Rico, Río Piedras. Se dedica este trabajo al gran mentor Frederick Scatena quien murió durante la finalización de este manuscrito. También se agradece a Alonso Ramírez y Eduardo Rosa-Molina de la Universidad de 
Puerto Rico-Río Piedras por proveer espacio de laboratorio. Este trabajo fue desarrollado dentro del Programa de Investigaciones Ecológicas a Largo Plazo en el Bosque de Luquillo, financiado por la Fundación Norteamericana para la Ciencias, y administrado conjuntamente por el Instituto Internacional de Dasonomía Tropical del Servicio Forestal de los Estados Unidos y la Universidad de Puerto Rico, Río Piedras. Finalmente se agradece a los estudiantes del internado de verano 2003 en la Estación de Campo "El Verde" de la Universidad de Puerto Rico financiado por la Fundación Nacional de Ciencias de los Estados Unidos, por su colaboración en los muestreos. Se agradece a los dos evaluadores anónimos y Alonso Ramírez y Pablo Gutiérrez por la organización de este número especial sobre "Ecología y Biomonitoreo de Macroinvertebrados de Agua Dulce en América Latina”. Contribución ELICE No. 13.

\section{RESUMEN}

Los gasterópodos Neritinidae de Puerto Rico presentan un ciclo de vida anfídromo y exhiben polimorfismo en los patrones de coloración de la concha. Se identificó que la oviposición aumenta río arriba conforme los individuos alcanzan mayores tamaños, a pesar de que la densidad poblacional disminuye. La frecuencia de siete fenotipos previamente establecidos se analizó en diez ríos de la isla durante dos años ( $\mathrm{n}=8751$ individuos). Debido a que el río Mameyes es el más conservado, se tomó como modelo para estudiar la variación a lo largo del río y entre hábitats durante tres años. El fenotipo de líneas axiales y puntos $(N$. virginea) fue el predominante en toda la isla y el fenotipo pateloide ( $N$. punctulata) fue el menos frecuente. Se observaron diferencias significativas de las frecuencias de los fenotipos (principalmente en los secundarios) entre ríos, a lo largo de ellos y entre hábitats. Esta variabilidad espacial puede responder a gradientes de salinidad y depredación. Se observó plasticidad fenotípica en algunos individuos.

Palabras clave: Neritina virginea, Neritina reclivata, Neritina punctulata, coloración de concha, variación fenotípica, polimorfismo.

\section{REFERENCIAS}

Abbott, R. T. (1974). American sea shells. Nueva York: Van Nostrand Reinhold.

Aguayo, C. G. (1966). Una lista de los moluscos terrestres y fluviales de Puerto Rico. Stahlia, 5, 1-17.
Andrews, E. A. (1935). Shell repair by the snail Neritina. Journal of Experimental Zoology, 70, 75-107.

Appleton, R. D., \& Palmer, A. R. (1988). Water-borne stimuli released by predatory crabs and damaged prey induce more predator-resistant shells in a marine gastropod. Proceedings of the National Academy of Sciences USA, 85, 4387-4391.

Bandel, K. (2001). The history of Theodoxus and Neritina connected with description and systematic evaluation of related Neritimorpha (Gastropoda). Mitteilungen aus dem Geologisch-Paläontologischen Institut der Universität Hamburg, 85, 65-164.

Blanco, J. F., \& Arroyave, A. (2009). Daños por depredación y tamaño de concha del caracol diádromo Neritina virginea (Gastropoda: Neritidae) en el Río Mameyes, Puerto Rico. Revista de Biología Tropical, 57, 1069-1080.

Blanco, J. F., \& Scatena, F. N. (2005). Floods, habitat hydraulics and upstream migration of Neritina virginea (Gastropoda: Neritidae) in Northeastern Puerto Rico. Caribbean Journal of Science, 41, 55-74.

Blanco, J. F., \& Scatena, F. N. (2006). Hierarchical contribution of river-ocean connectivity, water chemistry, hydraulics, and substrate to the distribution of diadromous snails in Puerto Rican streams. Journal of the North American Benthological Society, 25, 82-98.

Blanco, J. F., \& Scatena, F. N. (2007). The spatial arrangement of Neritina virginea (Gastropoda: Neritidae) during upstream migration in a split-channel reach. River Research and Applications, 23, 235-245.

Brookes, J. I., \& Rochette, R. (2007). Mechanism of a plastic phenotypic response: predator-induced shell thickening in the intertidal gastropod Littorina obtusata. Journal of Evolutionary Biology, 20, 1015-1027.

Brown, K. M., \& Quinn, J. F. (1988). The effect of wave action on growth in three species of intertidal gastropods. Oecologia, 75, 420-425.

Covich, A. P. (2010). Winning the biodiversity arms race among freshwater gastropods: competition and coexistence through shell variability and predator avoidance. Hydrobiologia, 653, 191-215.

Crandall, E. D. (1999). Early life history aspects of amphidromous neritid snails in Moorea, French Polynesia. Berkeley Science, 3, 98-102.

Díaz, J. M., \& Puyana, M. (1994). Moluscos del Caribe Colombiano. Santafé de Bogotá, Colombia: Colciencias-Fundación Natura-Invemar.

DeWitt, T. J. (1998). Costs and limits of phenotypic plasticity: tests with predator-induced morphology and life history in a freshwater snail. Journal of Evolutionary Biology, 11, 465-480.

Ekendahl, A. (1998). Colour polymorphic prey (Littorina saxatilis Olivi) and predatory effects of a crab 
population (Carcinus maenas L.). Journal of Experimental Marine Biology and Ecology, 222, 239-246.

Ford, J. I. (1979). Biology of a Hawaiian fluvial gastropod Neritina granosa Sowerby (Prosobranchia: Neritidae) (M.Sc. Thesis). University of Hawaii, Honolulu, Hawaii.

Grüneberg, H. (1976). Population studies on a polymorphic prosobrach snail (Clithon oualaniensis Lesson). Philosophical Transactions of the Royal Society of London, Biology, 275, 385-437.

Grüneberg, H. (1978). Microevolution in a polymorphic prosobranch snail (Clithon oualaniensis Lesson). Proceedings of the Royal Society of London, Biology, 200, 419-440.

Grüneberg, H. (1979). A search for causes of polymorphism in Clithon oualaniensis (Lesson) (Gastropoda: Prosobranchia). Proceedings of the Royal Society of London, Biology, 203, 379-386.

Grüneberg, H. (1982). Pseudopolymorphism in Clithon oualaniensis. Proceedings of the Royal Society of London, Biology, 216, 147-157.

Haynes, A. (1993). The gastropods in the streams and rivers of four islands (Guadalcanal, Makira, Malaita, and New Georgia) in the Solomon Islands. The Veliger, 36, 285-290.

Hazel, W. N., \& Johnson, M. S. (1990). Microhabitat choice in the land snail Thebapisana (Müller). Heredity, $65,449-454$

Hollander, J., \& Butlin, R. K. (2010). The adaptive value of phenotypic plasticity in two ecotypes of a marine gastropod. BMC Evolutionary Biology, 10, 1-7.

Hoverman, J. T., \& Relyea, R. A. (2007). How flexible is phenotypic plasticity? Developmental windows for trait induction and reversal. Ecology, 88, 693-705.

Humfrey, M. (1971). Sea shells of the West Indies. A guide to the marine molluscs of the Caribbean. Nueva York: Taplinger.

Johannesson, K., \& Ekendahl, A. (2002). Selective predation favouring cryptic individuals of marine snails (Littorina). Biological Journal of the Linnean Society, 76, 137-144.

Kano, Y. (2009). Hitchhiking behaviour in the obligatory upstream migration of amphidromous snails. Biology Letters, 5, 465-468.

Kemp, P., \& Bertness, M. D. (1984). Snail shape and growth rates: evidence for plastic shell allometry in Littorina littorea. Proceedings of the National Academy of Science USA, 81, 811-813.

McDowall, R. M. (2007). On amphidromy, a distint form of diadromy in aquatic organisms. Fish and Fisheries, $8,1-13$.

Manríquez, P., Lagos, N., Jara, M., \& Castilla, J. (2009). Adaptive shell color plasticity during the early ontogeny of an intertidal keystone snail. Proceedings of the National Academy of Science USA, 106, 16298-16303.

Meinhardt, H. (2003). The algorithmic beauty of sea shells. The Virtual Laboratory Series. Tübingen, Alemania: Springer.

Mestey-Villamil, V. (1979). Estudio biosistemático de la familia Neritidae (Mollusca: Archaeogastropoda) en Puerto Rico. M.Sc. Thesis, University of Puerto Rico, Río Piedras. Puerto Rico.

Minton, R. L., \& Gunderson, R. W. (2001). Puperita tristis (d'Orbigny, 1842) (Gastropoda, Neritidae) is an ecotype of Puperita pupa (Linnaeus, 1767). American Malacological Bulletin, 16, 13-20.

Minton, R. L., Norwood, A. P., \& Hayes, D. M. (2008). Quantifying phenotypic gradients in freshwater snails: a case study in Lithasia (Gastropoda: Pleuroceridae). Hydrobiologia, 605, 173-182.

Neumann, D. (1959). Experimentelle untersuchungen des farbenmusters der schale von Theodoxus fluviatilis. Zoologischer Anzeiger, 23, 152-156.

Palma, A., \& Steneck, R. (2001). Does variable coloration in juvenile marine crabs reduce risk of visual predation? Ecology, 82, 2961-2967.

Pettitt, C. (1975). A review of the predators of Littorina, especially those of L. saxatilis (Olivi) (Gastropoda: Prosobranchia). Journal of Conchology, 28, 343-357.

Pyron, M., \& Covich, A. (2003). Migration patterns, densities, and growth of Neritina punctulata snails in río Espíritu Santo and río Mameyes, Northeastern Puerto Rico. Caribbean Journal of Science, 39, 338-347.

Resh, V. H., Barnes, J. R., \& Craig, D. A. 1990. Distribution and ecology of benthic invertebrates in the Opunohu river catchment, Moorea, French Polynesia. Annales de Limnologie, 26, 195-214.

Schneider, D. W., \& Frost, T. M. (1986). Massive upstream migrations by a tropical freshwater neritid snail. Hydrobiologia, 137, 153-157.

Schneider, D. W., \& Lyons, J. (1993). Dynamics of upstream migration in two species of tropical freshwater snails. Journal of the North American Benthological Society, 12, 3-16.

Sepúlveda, R. D., Jara, C. G., \& Gallardo, C. S. (2012). Morphological analysis of two sympatric ecotypes and predator-induced phenotypic plasticity in Acanthina monodon (Gastropoda: Muricidae). Journal of Molluscan Studies, 78, 173-178.

Shigemiya, Y., \& Kato, M. (2001). Age distribution, growth and lifetime copulation frequency of a freshwater snail Clithon retropictus (Neritidae). Population Ecology, 43, 133-140.

Sokal, R. R., \& Rohlf, F. J. (1995). Biometry. San Francisco, CA: Freeman. 
Russell, H. D. (1941). The recent mollusks of the family Neritidae of the Western Atlantic. Bulletin of the Museum of Comparative Zoology, Harvard University, 88, 373-395.

Tan, S. K., \& Clements, R. (2008). Taxonomy and distribution of the Neritidae (Mollusca: Gastropoda) in Singapore. Zoological Studies, 47, 481-494.

Trussell, G. C., \& Nicklin, M. O. 2002. Cue sensitivity, inducible defenses, and trade-offs in a marine snail. Ecology, 83, 1635-1647.

Valdéz, M. F., \& Barrantes, R. (1980). Variabilidad geográfica y alozímica en Neritina latissima (Gastropoda: Neritidae) en Costa Rica. Revista de Biología Tropical, 28, 181-192.
Vermeij, G. J., \& Covich, A. P. (1978). Coevolution of freshwater gastropods and their predators. American Naturalist, 112, 833-843.

Vermeij, G. J. (1982). Unsuccessful predation and evolution. American Naturalist, 120, 701-720.

Warmke, G. L., \& Abbott, R. T. (1961). Caribbean sea shells. Pennsylvania: Livingston.

Zar, J. H. (1999). Biostatistical analysis. New Jersey: Prentice Hall.

Zettler, M. L., Frankowski, J. R., Bochert, \& Rohner, M. (2004). Morphological and ecological features of Theodoxus fluvialitis (Linnaeus, 1758) from Baltic brackish water and German freshwater populations. Journal of Conchology, 38, 305-316. 